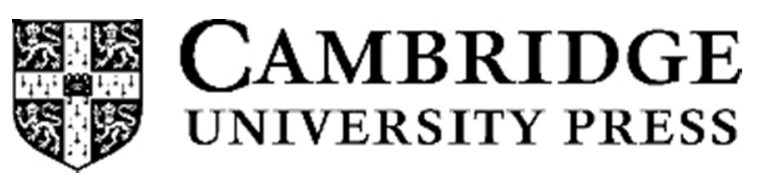

\title{
A novel approach to the examination of successful aging trajectories at the end of life
}

\begin{tabular}{|r|l|}
\hline Journal: & Canadian Journal on Aging/La Revue canadienne du vieillissement \\
\hline Manuscript ID & RCV-0591-E.R1 \\
\hline Manuscript Type: & Research Notes \\
\hline Keywords: & successful aging, end of life, growth mixture modelling \\
\hline \multicolumn{2}{|c}{} \\
\hline
\end{tabular}

\section{SCHOLARONE ${ }^{\text {M }}$ \\ Manuscripts}


SA at the end-of-life

A novel approach to the examination of successful aging trajectories at the end of life 
SA at the end-of-life

\begin{abstract}
:
Background: Traditional conceptualizations of successful aging (SA) have suggested that individuals experiencing functional decline at the end of life do not demonstrate SA. Despite the acknowledgement that SA is a heterogeneous life-course process, the granularity of previous analyses has been impeded by methodological and conceptual limitations.

Methods: A SA index was captured in a longitudinal population-based cohort study of individuals aged $\geq 75$ (Cambridge City over-75 Cohort Study) and examined longitudinally using growth mixture modelling (GMM) to identify groups with similar trajectories using decedents' $(n=1015)$ last completed interview and up to four previous data collection waves before death.
\end{abstract}

Results: GMM identified a three-class model, capturing high-functioning, no decline (HN), high-functioning, gradual decline (HG) and low-functioning, steep decline (LS) classes. Individuals in the HN class were significantly younger at death $(p<0.001)$ and at their last interview $(p<0.001)$, consisted of more men $(p<0.001)$, and were more likely to be married $(p<0.001)$ when compared to individuals in the HG and LS classes.

Conclusions: These results demonstrate the different ways in which individuals can experience SA at the end of life. The current study provides the framework for future research into life course processes of aging, with important implications for policy and practice. 
SA at the end-of-life

Introduction

The ways in which ageing has been conceptualised within a positive ageing framework has rapidly expanded from humble beginnings. In the early 1960 s Cumming \& Henry's Disengagement Theory (1961) was a popular - albeit, inherently negative perspective on ageing. Within this framework, to age well was to linearly retract from the activities of mid-life. However, in the first issue of The Gerontologist the late Robert Havighurst posited his Activity Theory (1961) , which took cues from positive psychology and in contrast to Disengagement Theory. However, even in these early days, Havighurst suggested that the operationalisation and articulation of successful aging (SA) would be problematic (Havighurst, 1961).

The MacArthur Foundation formed in 1984 under the leadership of John W. Rowe, with the intent to further articulate SA. A consortium of academics, geriatricians and gerontologists were gathered to critically examine the ways in which older adults age. From these meetings one of the most highly cited gerontological papers was produced: the Rowe \& Kahn model of SA (1987). In this model, a tripartite model of ageing was suggested, including high physical and cognitive functioning, low probability of disease and engagement (Rowe and Kahn, 1987). Alongside the many proponents of the Rowe \& Kahn (1987) model were those who, rightly, acknowledged oversights in the model. For example, Matilda Riley (1998) highlighted the notable absence of social situations in the model. These issues have been acknowledged by Rowe and Kahn and, where possible, improvements have been made.

In the burgeoning SA literature a number of areas remain that expand beyond the limitations of the Rowe \& Kahn model to fundamental conceptual and methodological 
SA at the end-of-life

limitations, notably with respect to the end of life (Cosco et al., 2013b). Additionally, the development of a consensus definition of SA has not occurred (Cosco et al., 2014a), which has had serious inhibitive effects on cross-study comparisons. Given the inability of SA models to articulate the heterogeneity of aging trajectories at the end of life (Cosco et al., 2013b), the implementation of more refined measures and methods of capturing SA across the life course is necessary (Kivimaki and Ferrie, 2011). The conceptual and methodological limitations of extant models have impeded this process, notably with respect to the selection of model components, the use of binary modelling procedures, the nature of the datasets used, and the statistical methods employed in the analysis of these data (Cosco et al., 2014c).

Over the past several decades the Rowe \& Kahn (1987) model of SA has been cited, examined and critiqued prolifically (Martinson and Berridge, 2015), collectively moving the field forward. In addition to quantitative analyses of SA, there have been many qualitative studies that have examined what SA means to the layperson. Unfortunately, many of the SA models implemented in quantitative studies have not benefitted from the input of laypersons (Jopp et al., 2014); theory-driven models greatly outnumber lay-informed models (Phelan et al., 2004). As a result, the relevance and impact of research conducted using these models to older individuals may be impeded.

Trajectories of ageing at the end of life may include some sort of functional decline, be it physical, cognitive or psychosocial. Unfortunately, the conceptual framework underpinning traditional models of SA cannot accommodate these declines in a meaningful way (Cosco et al., 2013b). Once an individual falls below a given threshold the individual is 
SA at the end-of-life

no longer deemed to be successful aging, inhibiting the examination of SA from a life course perspective.

In the modelling of SA, data-driven methods of examining the heterogeneity of SA trajectories have not been employed at the end of life. Other areas of research, such as studies of terminal decline in cognition, i.e the decline in cognitive functioning experienced immediately before death, have used modelling techniques similar to those employed in the current study, e.g. using time to death as the time metric, to examine decline at the end of life (Muniz-Terrera et al., 2011). However, these models focus on modelling change induced by proximity to death whereas SA is focused on acknowledging and articulating the heterogeneity of aging trajectories.

There is no consensus definition of SA. Further, laypersons and researchers are divided over which components should be included in these models (Cosco et al., 2014b). Recent systematic reviews of the literature reveal that laypersons generally suggest psychosocial components (Cosco et al., 2013a), whilst researchers suggest biomedical components (Cosco et al., 2014a). Further, researchers' conceptualizations have generally used binary modelling procedures suggesting that if an individual cannot sustain a high level of functioning across all physiological domains they can no longer be considered "successfully aging". Binary modelling of SA lacks the granularity to articulate the processes of aging, especially in the very old (e.g., persons aged 85 years and older) (Cosco et al., 2014c). Finally, the statistical procedures invoked to examine SA at the end of life generally posit absolute measures, e.g. thresholds of functioning, to capture heterogeneity in aging rather than relative measures, e.g. comparing functional capability between study participants. 
SA at the end-of-life

In order to longitudinally model SA across the life course, statistical procedures that align with the theoretical underpinnings of the SA model must be employed. The SA model was founded on the assertion that the aging process is heterogeneous (Rowe and Kahn, 1987); as such, methods that can examine the characteristics of individuals with similar longitudinal trajectories of a latent variable are advantageous. In contrast to variablecentred approaches, such as structural equation model and factor analysis, which focus on describing the relationships amongst variables, person-centred approaches, such as growth mixture modelling (GMM), describe relationships amongst individuals (Ding et al., 2007; Muthen and Muthen, 2000). Through the use of GMM it is possible to use a data-driven method to extract different classes of individuals with similar SA trajectories and to examine their characteristics at the end of life. To date, these procedures have not been employed at the end of life using a priori SA models that have been informed by layperson perspectives.

The current study aims to address the conceptual and methodological shortcomings of extant models of SA and to the heterogeneity of SA trajectories at the end of life using data in the Cambridge City over-75 Cohort Study (CC75C).

Methods

Study Participants

CC75C is a longitudinal population-based cohort study $(n=2610)$ founded in 1985 designed to measure the prevalence and incidence of dementia at very old age (Fleming et al., 2007). In the baseline wave of data collection, all men and women aged 75 years or older were sampled from five primary care practices, and one in three from a sixth practice in Cambridge (UK). A 95\% response rate was achieved. Follow-up interviews were 
SA at the end-of-life

conducted with surviving respondents at 3, 7, 9, 10, 13, 17, 21, 23, 25 and 28 years (Figure

1) capturing data on physical functioning, cognitive functioning, and psychosocial wellbeing.

SA Index

A SA index was created using components identified by operational definitions (Cosco et al., 2014a) and layperson perspectives (Cosco et al., 2013a) of SA. The SA index includes seven items: i) maintenance of interest ii) absence of loneliness iii) optimism iv) self-rated health v) cognitive functioning (i.e. Mini-Mental State Examination (MMSE)(Folstein et al., 1983)I vi) instrumental activities of daily living (IADLs)(Lawton and Brody, 1969), and vii) activities of daily living (ADLs)(Katz, 1983)s. Using a procedure similar to the Frailty Index (Searle et al., 2008), ordinal variables were assigned a value from 0 to 100 based on their reported level of functioning. For example, participants were asked "How do you feel about the future?", a response of "optimistic" would receive a score of 100, "empty expectations", a score of 50 and "pessimistic" a score of 0 . The one non-ordinal item, MMSE scores, were categorized using a similar procedure to Searle, et al. (2008), i.e. scores of 26-30 would receive a score of $100,22-25$ a score of $67,18-21$ a score of 33 , and 017 a score of 0 based on established cut-points for cognitive functioning. The scores from the seven constituent components were averaged to get a score between 0 and 100 , with increasing scores indicating more SA ( a more detailed description of the methods for constructing the SA index are available in Cosco, et al. (2015). In contrast to binary models that are unable to articulate the processes of aging with the necessary granularity needed for GMM (Cosco et al., 2014c), the indexing procedure provides a novel method of longitudinally quantifying SA.

Participant Classification 
SA at the end-of-life

Individuals who had died between each wave were identified using their date of death and date of interview. The SA Index was calculated for each individual at their last interview before death ( $\mathrm{t} 1$ ) and at up to three previous waves of data collection (t2-t4) (Figure 1). Variables necessary to create the SA index were not available in the baseline interview of the CC75C Study, therefore only scores from subsequent waves were used.

$<$ FIGURE 1 ABOUT HERE $>$

Covariates

Self-reported survey data was collected for all covariates. Socioeconomic status was indicated by the individual's employment before retirement according to the UK Registrar General's occupational classification and grouped into manual or non-manual, as per Lawlor, et al. (2004). Marital status was grouped into married or not married at the last wave of data collection. Education was captured using the individuals' self-reported age at which they left school.

Statistical Procedures

Latent variable modelling is a burgeoning area of interest with a ubiquitous presence in scientific research, allowing for the examination of unobserved, or latent, phenomena. As a result of advancements in statistical techniques and the increasing availability of relevant statistical software, latent variable modelling has become widely used in recent years (Loehlin, 2009). Latent variable modelling includes a number of 
SA at the end-of-life

methods, e.g. factor analysis, path analysis, structural equation modelling, and GMM, using observed variables to draw inferences about latent phenomena. Trajectories of SA were modelled in MPlus using GMM, a person-centred longitudinal latent variable analysis technique (Ding et al., 2007; Muthen and Muthen, 2000). In GMM models are estimated under the maximum likelihood estimation, with robust estimates under a missing at random assumption. The objective of GMM is to group similar individuals into separate classes, i.e. identify homogenous subpopulations within a larger heterogeneous population (Jung and Wickrama, 2008)(Muthen and Muthen, 2000), enabling the identification of disparate trajectories, for example of cognitive cognitive decline (Muniz-Terrera et al., 2009) or depression (Norton et al., 2011), when repeated-measure longitudinal datasets are employed, such as CC75C. The SA paradigm was founded on the acknowledgement that aging is a heterogeneous process (Rowe and Kahn, 1987); therefore, the theoretical underpinning of the SA model aligns closely with the person-centred approach used in GMM.

Due to issues of convergence in waves with $<10 \%$ of the original sample it was only possible to use four waves of data collection in the GMM analysis. Sex and age at death were included as covariates. Various permutations of the covariate relationships with the intercept and slope were used to find the best model fit once the appropriate number of trajectories had been identified.

The number of trajectories identified, i.e. model fit, was assessed using the Akaike Information Criteria (AIC) (Akaike, 1987), Bayesian Information Criterion (BIC) and sampleadjusted Bayesian Information Criterion (SABIC) (Nylund et al., 2007), with the lowest values on each measure was chosen to identify the best fitting model (Schwarz, 1978). Additionally 
SA at the end-of-life

the Lo-Mendell-Rubin Adjusted Likelihood Ratio Test (LMRLR) (Lo et al., 2001) and Parametric

Bootstrapped Likelihood Ratio Test (BLRT) (McCutcheon, 1987) were used to inform model selection, with models reaching significance rejecting the null hypothesis that the current number of classes are a better fit than fewer classes. Due to the nature of the model, which has been designed to fit non-normal data, the model must be interpreted within a theoretical framework as spurious classes may be identified (Bauer and Curran, 2003). Classification was assessed via evaluation of the entropy, an index that takes values between 0 and 1 with high values indicating a clear classification of individuals in classes (Celeux and Soromenho, 1996).

Demographic characteristics (sex, SES, education) across classes were compared using, $X^{2}$ tests for categorical variables and t-tests for continuous variables. These analyses were performed using Stata 12.

Missingness

The SA index calculates an average of components derived equally from layperson perspectives and researcher perspectives, therefore missing components' values would skew these data. Individuals that did not have complete data for the SA index were subject to listwise deletion during the creation of the index, i.e. if any component of the index was missing for a study participant, e.g. ADL score, he/she was excluded from the study.. Further, individuals that were still alive in the study were listwise deleted. Missingness at random for demographic variables, physical function (i.e. presence of disability in activities of daily living or instrumental activities of daily living), and cognitive function (Mini-Mental State Examination (Folstein et al., 1983) $\geq 27$ ) was assessed via $X^{2}$ tests or t-tests.

In GMM, models are estimated using maximum likelihood estimation, with robust estimates under a missing at random assumption (Jung and Wickrama, 2008). 
SA at the end-of-life

RESULTS

Of the 1180 participants interviewed at Survey 2, 1015 had SA index scores at t1, 361 at $\mathrm{t} 2,171$ at $\mathrm{t} 3$ and 65 at $\mathrm{t} 4$; mean number of waves participated in was 1.43 (SD 0.77 ). Individuals missing SA Index scores $(n=157)$ were significantly more likely to be women $\left(X^{2}\right.$ $=8.74, p=.003)$ less likely to be married $\left(X^{2}=16.16, p<.001\right)$ and to be older when they died $(t(1172)=-6.03, p<.001)$ and at their last interview $(t(1178)=-9.1911, p<.001)$, compared to included participants. No differences were observed between missing individuals' SES, education, and presence of cognitive/physical disability at Survey 2.

A three-class model provided the best fit to the data (Table 1) - capturing highfunction, no decline $(H N)(n=333,32.8 \%)$, high-function, gradual decline $(H G)(n=483$, 47.5\%), and low-function, steep decline (LS) $(n=199,19.6 \%)$ classes (Table 2$)$. This model had low AIC, BIC, and SABIC values, sufficient entropy, reasonable class sizes and the trajectories were theoretically sound. LMRLRT values suggested a four class model, however this model failed to converge.

<TABLE 1 ABOUT HERE>

<TABLE 2 ABOUT HERE>

The HN class exhibited virtually no decline, with individuals clustered at a high level of sustained functioning. The HG class had the highest initial level of functioning; however, in contrast to the HN class, exhibited a marked decline in SA index score towards death. The LS class exhibited the lowest initial functioning and the steepest decline in SA index score as individuals approached death (Figure 2).

<FIGURE 2 ABOUT HERE $>$ 
SA at the end-of-life

When compared with the HG class the HN class contained significantly more men, married participants, individuals that died earlier, and that had a longer interval between their last interview and death. When compared to the LS class the HN class contained significantly more men, married individuals, fewer manual labourers and individuals who died earlier and had a longer interval between the last interview and death. No differences were observed in educational attainment (i.e., school leaving age) across the three classes.

Discussion

The trajectories captured by the SA index scores demonstrate three separate end of life trajectories. Individuals in the HN class were predominately married men of high SES. These results suggest that individuals may have very different end of life trajectories within a SA framework, highlighting limitations in previous conceptualizations of SA through the use of novel indexing and modelling procedures.

Limitations in the creation of the SA index include missing values due to the listwise deletion of individuals that did not complete all components of the measure and due to attrition. Given that the index calculates an average value for all of the constituent components, individuals with missing data would not have a score comparable to those with complete data. Individuals excluded from the study differed from study participants in sex, marital status and age; however, no differences were identified in individuals SES, education, physical functioning and cognitive functioning at Survey 2, i.e. the first survey with all SA index components (Figure 1). The methods used in this longitudinal analysis employed time to death, rather than chronological age, as the time metric and use maximum likelihood estimation under a missing at random assumption, which are better able to accommodate attrition. Whether attrition due to death is truly missing at random is 
SA at the end-of-life

debatable and, therefore, a limitation of the current study. Further, individuals' information was included at their last wave of data collection. i.e. as close to death as possible. However, the interval between the last interview and the time of death varied across individuals, ranging from only a few days to several years.

The three identified trajectories differed not only in their intercepts, but also in their slopes, highlighting the different ways in which individuals can successfully age at the end of life. The HN class and HG classes began with a similar level of functioning, but had vastly different slopes of decline; the HG class declined at a much faster pace than the HN class, which experienced very little decline. This is an important relationship to explore, particularly with regards to protective factors that permit individuals in the $\mathrm{HN}$ to maintain their high level of functioning. Through the examination of why individuals in the HG class started at the same level of functioning, but declined much more rapidly, perhaps this decline can be staved off or attenuated. Further, examining modifiable lifestyle behaviours and social mechanisms that explain the differences in slope between HG and LS classes will provide important insights to the end of life process.

The trajectories modelled in the current study benefitted from the use of a metric of SA that has improved granularity over binary models and longitudinal latent variable modelling techniques that allow the identification of different classes of SA. Previous studies have faced conceptual challenges in the creation of SA models that are relevant to researchers and older adults; these issues have been addressed through the use of an index that is informed by systematic reviews of layperson perspectives and operational definitions of SA. Methodologically, extant models have not been able to articulate SA due to the use of binary models, which have inhibited the accommodation of decline. Through the use of 
SA at the end-of-life

time to death as the time metric, individuals' data from the end of life can be modelled using latent variable modelling procedures, combatting these issues. These results have important implications for further research, highlighting alternative and novel means with which to model processes, such as SA, using indexing procedures and longitudinal latent variable modelling. Further, these results highlight the potential for important research possibilities into the provision of resources and care in the dying process. If these procedures can elucidate different SA trajectories towards the end of life, the relationship between modifiable lifestyle behaviours and these classes can be identified, with the potential for implementing policies and interventions to facilitate these outcomes.

As highlighted by the trajectories identified via GMM, SA is not an all or nothing state. Rather, there are many ways in which individuals experience SA and the dying process. Further work investigating risk and protective factors for SA will have important implications for policy implementation and intervention trials focused on the fostering of better biopsychosocial functioning at the end of life.

The current study provides a novel framework and method with which to examine heterogeneous trajectories of SA at the end of life. These results provide a step forward in the conceptualization and examination of the disparate ways in which individuals can age well, highlighting the potential for future research to examine factors that foster more positive end of life experiences. Further research is required to unpick the specific mechanisms behind these different SA trajectories; however, the current study provides the conceptual and methodological framework for the advancement of these areas of research into the processes of SA at the end of life. 
SA at the end-of-life

\section{References}

Akaike, H. (1987). Factor-Analysis and Aic. Psychometrika, 52, 317-332.

Bauer, D. and Curran, P. (2003). Distributional assumptions of growth mixture models: implications for overextraction of latent trajectory classes. Psychological methods, 8, 338363.

Celeux, G. and Soromenho, G. (1996). An entropy criterion for assessing the number of clusters in a mixture model. Journal of Classification, 13, 195-212.

Cosco, T. D., Prina, A. M., Perales, J., Stephan, B. and Brayne, C. (2013a). Lay perspectives of successful ageing: a systematic review and meta-ethnography. Bmj Open, 3, e002710. Cosco, T. D., Prina, A. M., Perales, J., Stephan, B. and Brayne, C. (2014a). Operational definitions of successful aging: A systematic review. International Psychogeriatrics, 26, 373381.

Cosco, T. D., Prina, A. M., Perales, J., Stephan, B. and Brayne, C. (2014b). Whose "successful ageing"? Lay- and researcher-driven conceptualisations of ageing well European Journal of Psychiatry, 28, 124-130.

Cosco, T. D., Stephan, B. and Brayne, C. (2013b). Deathless models of aging and the importance of acknowledging the dying process. Canadian Medical Association Journal, 185, 751-752.

Cosco, T. D., Stephan, B. and Brayne, C. (2014c). (Unsuccessful) binary modeling of successful aging in the oldest-old adults: a call for continuum-based measures. Journal of the American Geriatrics Society, 62, 1597-1598.

Cosco, T. D., Stephan, B. C. and Brayne, C. (2015). Validation of an a priori, index model of successful aging in a population-based cohort study: the successful aging index. Int Psychogeriatr, 27, 1971-1977. 
SA at the end-of-life

Cumming, E. and Henry, W. (1961). Growing Old. New York: Basic.

Ding, J., et al. (2007). Effects of birth cohort and age on body composition in a sample of community-based elderly. American Journal of Clinical Nutrition, 85 (2), 405-410.

Fleming, J., Zhao, E., O'Connor, D., Pollitt, P. A. and Brayne, C. (2007). Cohort profile: the Cambridge City over-75s Cohort (CC75C). International Journal of Epidemiology, 36, 40-46. Folstein, M., Robins, L. and Helzer, J. (1983). The Mini-Mental State Examination. Archives of general psychiatry, 40, 812.

Havighurst, R. (1961). Successful aging. The Gerontologist, 1, 8-13.

Jopp, D. S., Wozniak, D., Damarin, A. K., De Feo, M., Jung, S. and Jeswani, S. (2014). How Could Lay Perspectives on Successful Aging Complement Scientific Theory? Findings From a U.S. and a German Life-Span Sample. The Gerontologist.

Jung, T. and Wickrama, K. (2008). An introduction to latent class growth analysis and growth mixture modeling. Social and Personality Psychology Compass, 2, 302-317.

Katz, S. (1983). Assessing self-maintenance: activities of daily living, mobility, and instrumental activities of daily living. Journal of the American Geriatrics Society, 31, 721-727. Kivimaki, M. and Ferrie, J. E. (2011). Epidemiology of healthy ageing and the idea of more refined outcome measures. Int J Epidemiol, 40, 845-847.

Lawlor, D. A., Smith, G. D. and Ebrahim, S. (2004). Association between childhood socioeconomic status and coronary heart disease risk among postmenopausal women: findings from the British Women's Heart and Health Study. American journal of public health, 94, 1386-1392.

Lawton, M. and Brody, E. (1969). Assessment of older people: self-maintaining and instrumental activities of daily living. The Gerontologist, 9, 179-186. 
SA at the end-of-life

Lo, Y., Mendell, N. and Rubin, D. (2001). Testing the number of components in a normal mixture. Biometrika, 88, 767-778.

Loehlin, C. (2009). Latent variable models: An introduction to factor, path, and structural equation analysis. New Jersey: Lawrence Erlbaum Associates, Inc. .

Martinson, M. and Berridge, C. (2015). Successful aging and its discontents: a systematic review of the social gerontology literature. The Gerontologist, 55, 58-69.

McCutcheon, A. (1987). Latent class analysis. Newbury Park, CA: Sage.

Muniz-Terrera, G., Matthews, F., Dening, T., Huppert, F. A. and Brayne, C. (2009). Education and trajectories of cognitive decline over 9 years in very old people: methods and risk analysis. Age and ageing, 38, 277-282.

Muniz-Terrera, G., Matthews, F. E., Stephan, B., Brayne, C. and Group, C. C. C. (2011). Are terminal decline and its potential indicators detectable in population studies of the oldest old? International Journal of Geriatric Psychiatry, 26, 584-592.

Muthen, B. and Muthen, L. (2000). Integrating person-centered and variable-centered analyses: growth mixture modeling with latent trajectory classes. Alcoholism, clinical and experimental research, 24, 882-891.

Norton, S., Sacker, A., Young, A. and Done, J. (2011). Distinct psychological distress trajectories in rheumatoid arthritis: findings from an inception cohort. J Psychosom Res, 71, 290-295.

Nylund, K. L., Asparoutiov, T. and Muthen, B. O. (2007). Deciding on the number of classes in latent class analysis and growth mixture modeling: A Monte Carlo simulation study. Structural Equation Modeling-a Multidisciplinary Journal, 14, 535-569. 
SA at the end-of-life

Phelan, E., Anderson, L., LaCroix, A. and Larson, E. (2004). Older adults' views of "successful aging"--how do they compare with researchers' definitions? Journal of the American Geriatrics Society, 52, 211-216.

Riley, M. W. (1998). Successful aging. The Gerontologist, 38, 151.

Rowe, J. and Kahn, R. (1987). Human aging: usual and successful. Science, 237, 143-149.

Schwarz, G. (1978). Estimating Dimension of a Model. Annals of Statistics, 6, 461-464.

Searle, S., Mitnitski, A., Gahbauer, E., Gill, T. M. and Rockwood, K. (2008). A standard procedure for creating a frailty index. Bmc Geriatrics, 8, 24. 
Table 1: Model selection criteria

$\begin{array}{cccccccr}\text { Class } & \text { AIC } & \text { BIC } & \text { SABIC } & \text { Entropy } & \begin{array}{c}\text { Smallest Class } \\ \text { (\% of sample) }\end{array} & \text { LMRLRT } & \text { BLRT } \\ & & & & & & & \\ 2 & 11105.707 & 11179.5447 & 11131.906 & 0.58 & 0.33 & <0.001 & <0.001 \\ 3 & 10993.565 & 11101.863 & 11031.989 & 0.61 & 0.20 & <0.001 & <0.001 \\ 4^{*} & 10963.828 & 11116.43 & 11017.972 & 0.64 & 0.07 & 0.23 & <0.001\end{array}$

Notes

* Did not converge; AIC: Akaike Information Criteria; BIC: Bayesian Information Criteria; SABIC: Sample-adjusted Bayesian Information Criteria; LMRLRT: Lo-Mendell-Rubin Adjusted Likelihood Ratio Test; BLRT: Parametric Bootstrapped Likelihood Ratio Test 
Table 2: SA trajectory characteristics

\begin{tabular}{|c|c|c|c|c|c|c|c|c|c|c|c|c|c|c|}
\hline & & \multirow[b]{2}{*}{$\mathrm{n}$} & \multirow[b]{2}{*}{ Intercept } & \multirow[b]{2}{*}{ Slope } & \multirow{2}{*}{$\begin{array}{c}\text { Women } \\
\text { (\%) }\end{array}$} & \multirow{2}{*}{$\begin{array}{c}\text { Married } \\
\text { (\%) }\end{array}$} & \multirow{2}{*}{$\begin{array}{l}\text { Manual } \\
\text { Work } \\
\text { (\%) }\end{array}$} & \multirow{2}{*}{$\begin{array}{l}\text { Age left } \\
\text { school } \\
\text { (years) } \\
\text { mean }\end{array}$} & \multicolumn{2}{|c|}{$\begin{array}{l}\text { Age at last } \\
\text { interview }\end{array}$} & \multicolumn{2}{|c|}{ Age at death } & \multicolumn{2}{|c|}{$\begin{array}{l}\text { Time between } \\
\text { last interview } \\
\text { and death } \\
\text { (years) }\end{array}$} \\
\hline & & & & & & & & & mean & SD & mean & SD & Mean & SD \\
\hline \multirow[t]{2}{*}{ Total } & & 1015 & & & 63.94 & 31.72 & 61.36 & 14.80 & 84.60 & 4.32 & 89.47 & 5.04 & 2.73 & 2.29 \\
\hline & $\mathrm{HN}^{+}$ & 333 & 95.64 & -0.31 & 48.05 & 50.75 & 56.31 & 14.98 & 82.94 & 3.59 & 88.29 & 4.90 & 3.03 & 2.74 \\
\hline \multirow[t]{2}{*}{ Class } & HG & 483 & 96.98 & -2.47 & $68.94 * * *$ & $23.86 * * *$ & 63.11 & 14.74 & $85.03 * * *$ & 4.24 & $89.88^{* * *}$ & 4.96 & $2.68^{*}$ & 2.07 \\
\hline & LS & 199 & 90.04 & -4.25 & $78.39 * * *$ & $18.78^{* * *}$ & $65.63 *$ & 14.65 & $86.29 * * *$ & 4.70 & $90.47 * * *$ & 5.10 & $2.37^{* *}$ & 1.91 \\
\hline
\end{tabular}

${ }^{\dagger}$ Comparison group; * $p<0.05,{ }^{* *} p<0.01,{ }^{* * *} p<0.001$

Key:

HN: high-function, no decline; HG: high-function, gradual decline; LS: low-function, steep decline 


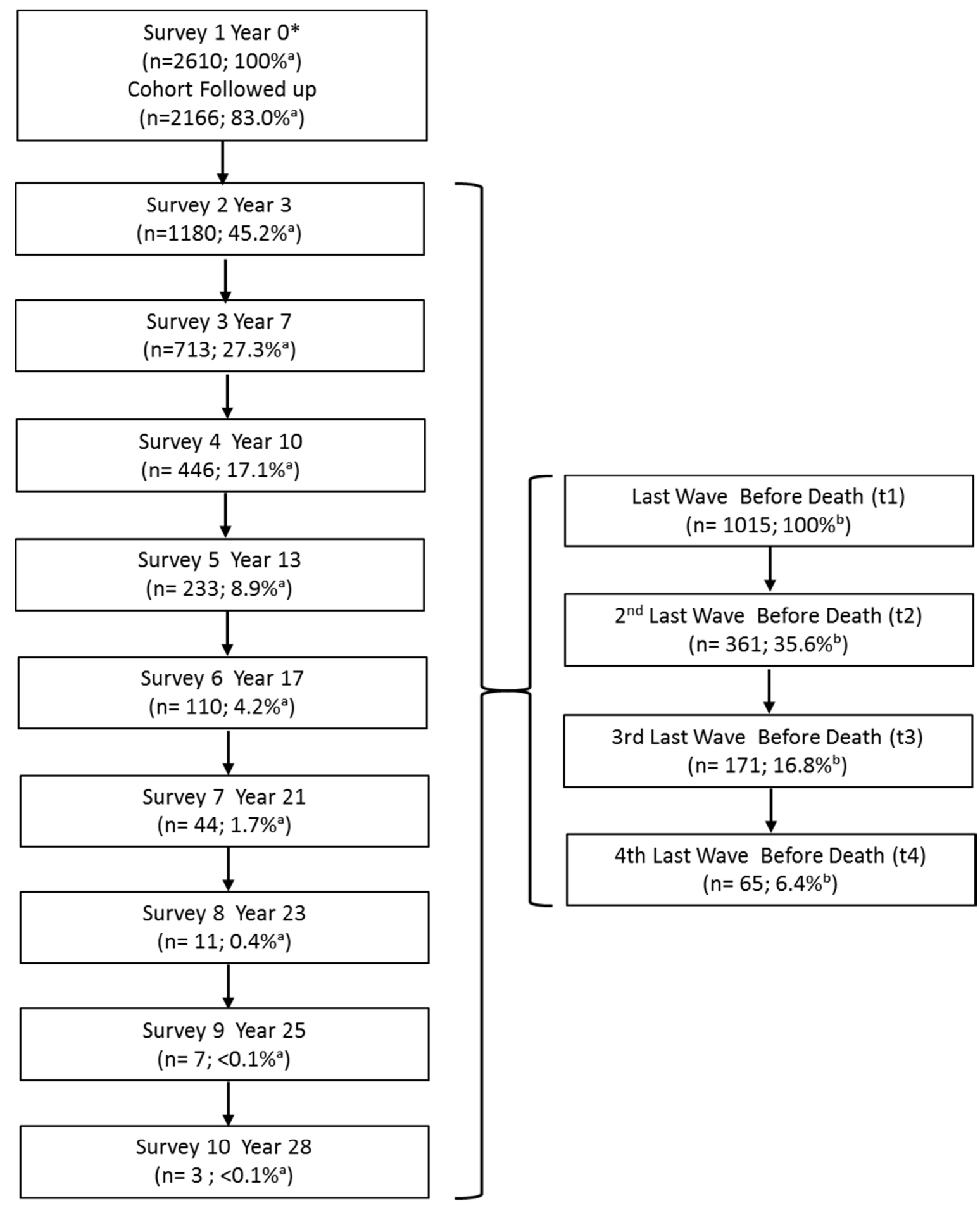

* Did not include components for SA Index a Percentage of original sample ${ }^{b}$ Percentage of sample in the present study 


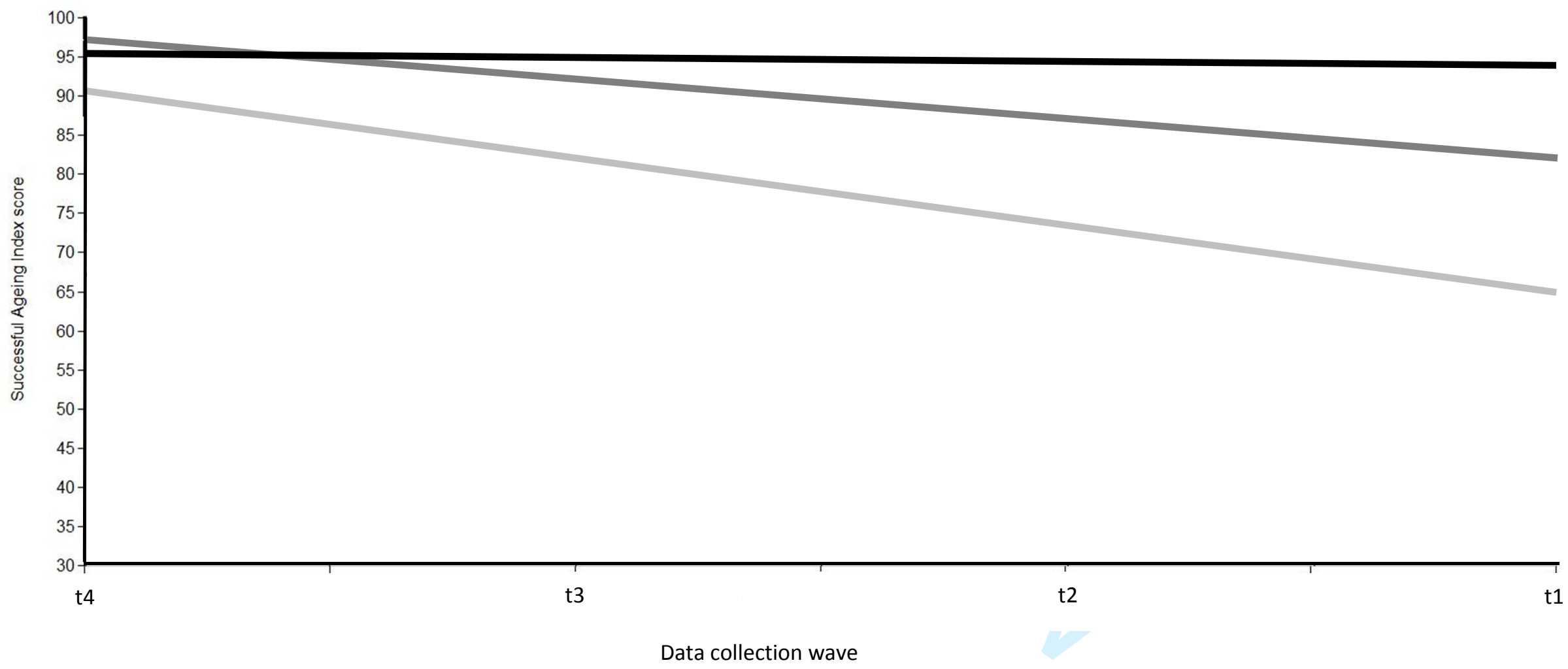

Figure 2: Observed values and estimated mean trajectories 
Blue line: Estimated mean trajectory for HN class; Red line: Estimated mean trajectory for HG class; Green line: Estimated mean trajectory for LS class; Black lines: Observed values for all individuals; t4-1: data collection waves from death, i.e. t1 is the last data collection wave before death 